\title{
Internet of Things (IOT) for Railways
}

\author{
Perumallapalli Krishna
}

\begin{abstract}
The biggest problems of the Indian railways are accidents and more delay time. The Internet of Things (IOT) can be used to reduce the effect of above two problems. Many accidents occur because of un-attended railway gates, over speed and improper signaling. The information of trains is not so clear to the railway stations, which leads to delay in trains. In this paper some solutions are proposed, to make the railways better, like automatic gate control, train tracking using Global Positioning System (GPS) and track switching. This can be achieved by IOT along with database management system (DBMS) and Radio Frequency Identification (RFID) technology. Each train is tracked using RFID and information is updated into the DBMS.
\end{abstract}

Keywords : IOT, automatic gate control, GPS, DBMS and RFID.

\section{INTRODUCTION}

Railways being the cheapest mode of transportation are preferred over all the other means. When we go through the daily newspapers we come across many railway accidents occurring at unmanned railway crossings obstacle on track system. This is mainly due to the carelessness in manual operations or lack of workers. This paper is proposed to make the railways smart. The system proposed in this paper is only the basic idea of smart railways but so many features need to be included for betterment and reliability of the system [1]. The backbone of the system is database management system (DBMS). The entire information of all trains like train number, train name, source and destination stations, train timings (schedule like halting stations and corresponding halting time) and type of train i.e express or passenger. Even the trains itself can be made smart by adding some features. Recently India witnessed many accidents due to derailing which led to loss of human and money. If the train is able to understand the track situation and take decision, the accidents can be minimized.

\subsection{System design}

For making railways better, Internet being powerful tool to connect one device to the other and access the information faster using present technology, is used to connect all the systems. Since the railway system will become more complex than existing, after connecting all the required devices and technology, in this paper a small prototype is proposed. To make the system more efficient, a high speed internet is required, along with the entire required Information Technology (IT) infrastructure. Each train is allotted a RFID tag which contains the entire information of the train [2]. To get the information from RFID tag, a RFID reader is placed, at

Revised Manuscript Received on September 22, 2019

* Correspondence Author

Perumallapalli Krishna, RGUKT, Nuzvid-521202, Andhra Pradesh, India

each station. The RFID tag contains an electronic chip which carries the information of the train. The following are the details of train stored in RFID tag,

- Train number

- Train name

- From and To station

- Number of stations passes by

- Number of halting stations along with duration of halting and train schedule

- Class of train (like express, passenger etc)

At each station, when the train passes, the RFID reader reads the information and updates to the DBMS via internet. The same information can be used to track the train or a Global positioning System is placed to track the exact location of train using longitude and latitude. Anyone with internet can access the live train status more accurately using the system proposed.

For controlling gate automatically Infrared (IR) sensors are used [3]. When a train approaches a particular railway crossing from either side, the sensors which are placed at a certain distance, detects the train and accordingly controls the gate with the help of a motor. Also an indicator light is provided to alert the motorists about the train. Once the train crosses the other side IR sensor, gate will be opened for motorists. At each railway crossing the information of train is updated and stored in the required format for future purpose.

Track switching is proposed to avoid accidents when two trains travelling on the same track in opposite direction. This involves lot of infrastructure development and better controlling mechanism. The entire information either gate control or track switching is updated every time to DBMS and keep record for future purpose. The structure of the proposed system is shown in Fig.1.

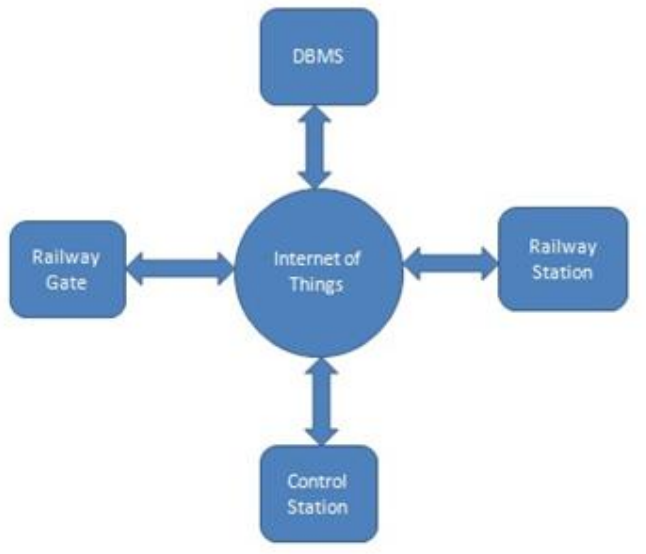

Fig.1 Block diagream of Railways using IOT

\subsection{Automatic Gate control and Track switching}

The block diagram of automatic gate control and track switching are shown in Fig.2 


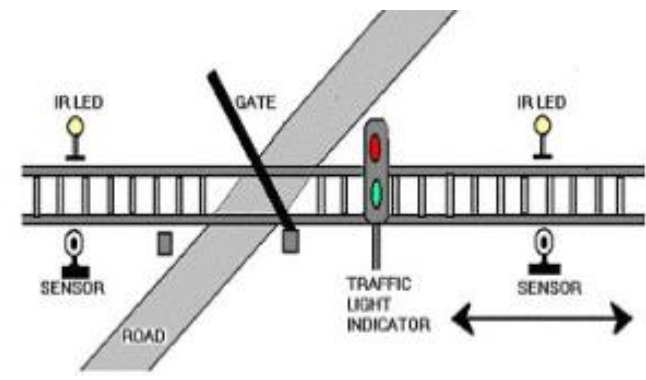

Figure 2.(a) Gate control

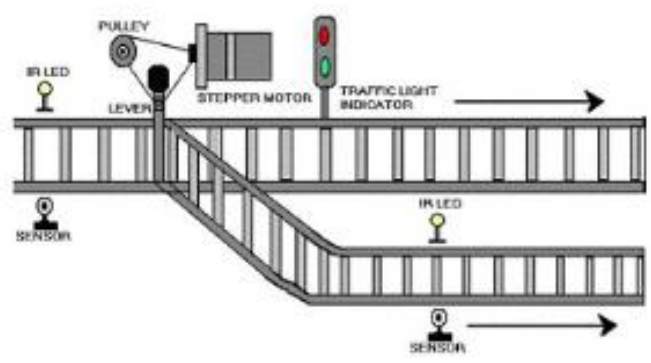

Figure 2. (b) Track switching

In the proposed system ARDUINO is used for generating controlling signals. The proposed system is tested with one train for gate control and two trains for track switching. As a train approaches, sensor detects and control signal is generated to control the motor, the gate is pulled up to ensure safety and avoid accidents [4]. The signal about the departure is sent to the ARDUINO, with IR sensor at the other end, which in turn operates the motor and opens the gate.

Using the same principle as that of gate control, the concept of automatic track switching is developed [5]. The situation of two trains coming opposite is a rare case but when it occurs, the system developed in this paper acts upon and diverts the one of the train to the other track to avoid accidents. This is possible if second track is available. The entire information of track and gate are stored in DBMS and accessed whenever necessary. After taking action, the information will be updated for future purpose. The information stored in the DBMS will be analyzed from time to time for making the system further better.

\subsection{Train information Display and Tracking}

As each train carries a RFID tag, which uses radio frequency electromagnetic fields to identify objects when they come close to a reader, data included in the electronic chip will be collected by the reader or interrogator [6]. The readers are placed away from the station. RFID tags generally feature an electronic chip with an antenna in order to pass information onto the reader. The proposed system is shown in Fig.3

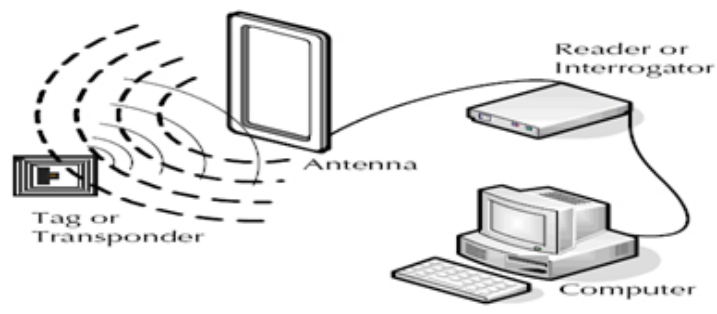

Figure 3 RFID reader
After each RFID tag read by the reader, information will be sent to control station and DBMS to act upon the necessary action. The same information can be used for train tracking but to make it more accurate tracking, especially for express trains, GPS is used. At the stations and crossings the information is displayed on Liquid Crystal Display (LCD).

\subsection{Accident alert}

The GPS module provides coordinates and the accelerometer values will be recorded continuously to detect any malfunction in engine. The system will give an alert message to the concerned person and control station. The accident alert system will also examine the track and alert the train driver.

\section{RESULTS AND CONCLUSION}

The model is tested with two trains and experimented with all the above proposed tasks. The model proposed reduces the faults in the existing railway system like more delay time, accidents and live train tracking. This model, if implemented, improves railways efficiency and reduces loss of people and income. The accidents are avoided at places where there is no person managing the railway crossing gates. Since the entire information is updated time to time, the trains and tracks will be analyzed for faults and performance, well in advance.

\section{ACKNOWLEDGEMENT}

The author acknowledges the support of Rajiv Gandhi University of Knowledge Technologies, Nuzvid, Andhra Pradesh, India for its support and encouragement.

\section{REFERENCES}

1. Hemanth T.Mahajan,Ankita D.Mosamkar,Jyoti J.Patil,Divya D.Patil,Renuka L.Patil,”A Review paper: Smart Train System Using IOT", Journal of Automation and Automobile Engineering,Volume 3,Isssue1,pp.1-3

2. $\mathrm{Pb}$ Agus Ristono*,"Design Of Reliable And Efficient Manchester Carry Chain Adder Based 8-Bit Alu For High Speed Applications",Journal Of VLSI Circuits And Systems, 1 (01), 1-4,2019

3. NHK K. ISMAIL*,'Estimation Of Reliability Of D Flip-Flops Using Mc Analysis",

4. Journal of VLSI Circuits And Systems 1 (01), 10-12,2019

5. Saifuddin mahmud, Ishtiag Reza Emon, Md.Mohaimin Billah, "Automated Railway gate Controlling System", International Journal of Computer trends and and Technology, Volume 27,Number 1 September 2015

6. Subrata Biswas, Rafiul Hoque Bhuiyan, samiul Hoque, Robiul Hasan, Tanzila Nusrat Khan, "Pressure sensed fast Response Anti-Collision system for Automated Railway gate Control", American Journal of Engineering Research, Volume 2, Issue 11, 2013, pp.163-173

7. K.ajith Theja, Dr.M.Kumaresan, Dr.K.Senthil Kumar,"Automated Unmanned Railway Level Crossing System Using WSN", International Journal of Innovative Research in Computer and Communication Engineering, Volume 3, Issue 11, November 2015, pp.11382-1. 